\title{
A General Iterative Algorithm with Strongly Positive Operators for Strict Pseudo-Contractions
}

\author{
Wei $X u^{1}$ and Yuanheng Wang ${ }^{2}$ \\ ${ }^{1}$ Tongji Zhejiang College, Zhejiang 314000, China \\ ${ }^{2}$ Department of Mathematics, Zhejiang Normal University, Zhejiang 321004, China \\ Correspondence should be addressed to Yuanheng Wang; wangyuanheng@yahoo.com.cn
}

Received 6 November 2012; Accepted 2 February 2013

Academic Editor: Claudia Timofte

Copyright (c) 2013 W. Xu and Y. Wang. This is an open access article distributed under the Creative Commons Attribution License, which permits unrestricted use, distribution, and reproduction in any medium, provided the original work is properly cited.

\begin{abstract}
This paper deals with a new iterative algorithm $\left\{x_{n}\right\}$ with a strongly positive operator $A$ for a $k$-strict pseudo-contraction $T$ and a non-self-Lipschitzian mapping $S$ in Hilbert spaces. Under certain appropriate conditions, the sequence $\left\{x_{n}\right\}$ converges strongly to a fixed point of $T$, which solves some variational inequality. The results here improve and extend some recent related results.
\end{abstract}

\section{Introduction}

Let $C$ be a closed convex subset of Hilbert space $H$ with inner product $\langle\cdot, \cdot\rangle$ and norm $\|\cdot\|, T: C \rightarrow H$ be a nonlinear mapping. The fixed point set of $T$ is denoted by $\operatorname{Fix}(T)$; that is, $\operatorname{Fix}(T)=\{x \in C, T x=x\}$. Fixed point problem is very general in the sense that it includes, as spacial cases, optimization problems, variational inequalities, minimax problems, the Nash equilibrium problem in noncooperative games, and others.

Recall that a mapping $T: C \mapsto C$ is said to be nonexpansive if $\|T x-T y\| \leq\|x-y\|$ for all $x, y \in C$. A mapping $T: H \mapsto H$ is said to be strongly positive, if there exists a constant $\gamma>0$ such that $\langle A x, x\rangle \geq$ $\gamma\|x\|^{2}$ for all $x \in H$. In 2000, Moudafi [1] investigated the fixed point problem of nonexpansive mapping with viscosity approximation method. Let $f$ be a contraction on $H$; that is, there exists a constant $\alpha \in(0,1)$ such that $\|f(x)-f(y)\| \leq$ $\alpha\|x-y\|$ for all $x, y \in C$; define a sequence $\left\{x_{n}\right\}$ by

$$
x_{n+1}=\alpha_{n} f\left(x_{n}\right)+\left(1-\alpha_{n}\right) T x_{n}, \quad \forall n \geq 0,
$$

where $x_{0}$ is an arbitrary starting point in $H$ and $\left\{\alpha_{n}\right\}$ is a sequence in $(0,1)$. In $2004 \mathrm{Xu}$ [2] proved that if the parameter $\left\{\alpha_{n}\right\}$ satisfies some approximate conditions, the sequence $\left\{x_{n}\right\}$ generated by (1) converges strongly to not only a fixed point of $T$ but also the unique solution $x^{*}$ of the variational inequality

$$
\left\langle(I-f) x^{*}, x-x^{*}\right\rangle \geq 0, \quad \forall x \in \operatorname{Fix}(T) .
$$

In 2010, Tian [3] considered a general hybrid steepestdescent method:

$$
x_{n+1}=\alpha_{n} \gamma f\left(x_{n}\right)+\left(I-\mu \alpha_{n} F\right) T x_{n}, \quad \forall n \geq 0,
$$

where $F$ is a Lipschitzian and strongly monotone operator. Under certain conditions, he proved that the sequence $\left\{x_{n}\right\}$ generated by (3) converges strongly to the unique solution $x^{*}$ of the variational inequality

$$
\left\langle(\gamma f-\mu F) x^{*}, x-x^{*}\right\rangle \leq 0, \quad \forall x \in \operatorname{Fix}(T) .
$$

On the other hand, Marino and $\mathrm{Xu}$ [4] introduced the following iterative scheme:

$$
x_{n+1}=\alpha_{n} \gamma f\left(x_{n}\right)+\left(I-\alpha_{n} A\right) T x_{n}, \quad \forall n \geq 0,
$$

where $A$ is a strongly positive bounded linear operator. It was proven that under certain conditions on the parameters, the sequence $\left\{x_{n}\right\}$ generated by (5) converges strongly to the unique solution $x^{*}$ of the variational inequality

$$
\left\langle(\gamma f-A) x^{*}, x-x^{*}\right\rangle \leq 0, \quad \forall x \in \operatorname{Fix}(T) .
$$


It is well known that a typical convex minimization is that of minimizing a quadratic function on the sets of the fixed points of a nonexpansive mapping:

$$
\underset{x \in \operatorname{Fix}(T)}{\operatorname{Min}} \frac{1}{2}\langle A x, x\rangle-\langle x, b\rangle
$$

where $b$ is a given point of $H$. The solution $x^{*}$ is also the optimality condition for the minimization problem

$$
\underset{x \in \operatorname{Fix}(T)}{\operatorname{Min}} \frac{1}{2}\langle A x, x\rangle-h(x),
$$

where $h(x)$ is a potential function for $\gamma f$; that is, $h^{\prime}(x)=$ $\gamma f(x), \forall x \in H$. Some authors investigated each iterative method for nonexpansive mappings for solving convex minimization problems and got some convergence results; see, for example [5-7].

In 2011, Ceng et al. [8] introduced a general iterative algorithm with strongly positive operators for nonexpansive mappings:

$$
\begin{aligned}
& y_{n}=\left(I-\mu \alpha_{n} F\right) T x_{n}+\alpha_{n} \gamma f\left(x_{n}\right), \\
& x_{n+1}=\left(I-\beta_{n} A\right) T x_{n}+\beta_{n} y_{n}, \quad \forall n \geq 0
\end{aligned}
$$

and proved that under certain conditions on the parameters the sequence $\left\{x_{n}\right\}$ generated by (9) converges strongly to a fixed point $x^{*}$ of $T$, which also solves the variational inequality

$$
\left\langle(I-A) x^{*}, x-x^{*}\right\rangle \leq 0, \quad \forall x \in \operatorname{Fix}(T) .
$$

Recently the problems of the approximation of the common fixed points of nonexpansive mappings were extended to the case of a family of finite or infinite pseudo-contractions; see, for example, [9-11].

Motivated and inspired by the above research works, we consider some fixed point problems with non-self mappings and introduce a new general iterative algorithm with strongly positive operators for $k$-strict pseudo-contractions which is a wider map class then the nonexpansive map class

$$
\begin{aligned}
& y_{n}=P_{C}\left[\alpha_{n} \tau S x_{n}+\left(I-\mu \alpha_{n} F\right) T x_{n}\right], \\
& x_{n+1}=\left(I-\left(\gamma_{n}+\delta_{n}\right) A\right) y_{n}+\gamma_{n} x_{n}+\delta_{n} T x_{n},
\end{aligned}
$$

$\forall n \geq 0$,

where $P_{C}: H \rightarrow C$ is the metric projection, $S: C \mapsto H$ is a non-self-Lipschitzian mapping, $T: C \mapsto C$ is a $k$-strict pseudo-contraction, and $A: C \mapsto C$ is a strongly positive bounded linear operator. Under certain conditions on the parameters, we prove that the sequence $\left\{x_{n}\right\}$ generated by (11) converges strongly to a fixed point $x^{*}$ of $T$, which solves the variational inequality

$$
\left\langle(I-A) x^{*}, z-x^{*}\right\rangle \leq 0, \quad \forall z \in \operatorname{Fix}(T) .
$$

\section{Preliminaries}

In this section, we recall some useful definitions and lemmas for the proof of the main results.
Definition 1. A mapping $T: C \mapsto C$ is said to be $L$ Lipschitzian, if there exists a constant $L>0$ such that

$$
\|f(x)-f(y)\| \leq L\|x-y\| \quad \forall x, y \in C .
$$

A mapping $T: C \mapsto C$ is said to be $k$-strict pseudocontraction, if there exists a constant $k \in[0,1)$ such that

$$
\begin{array}{r}
\|T x-T y\|^{2} \leq\|x-y\|^{2}+k\|(I-T) x-(I-T) y\|^{2}, \\
\forall x, y \in C .
\end{array}
$$

It is clear that a Lipschitzian map is a contractive map when $0<L<1$ and is a nonexpansive map when $L=1$. If $k=$ 0 ; then a $k$-strict pseudo-contraction map is a nonexpansive map.

Definition 2. A mapping $P_{C}: H \mapsto C$ is said to be the metric projection, if for any $x \in H$, there exists a unique nearest point in $C$ denoted by $P_{C} x$ such that

$$
\left\|x-P_{C} x\right\| \leq\|x-y\|, \quad \forall y \in C .
$$

And it is well known that if $C$ is a nonempty closed convex subset of $H$, then the $P_{C}$ exists (e.g., see [12]).

Lemma 3 (see [13]). Let $x \in H$ and $z \in C$ be any points. There holds

$$
\left\langle P_{C} x-P_{C} y, x-y\right\rangle \geq\left\|P_{C} x-P_{C} y\right\|^{2}, \quad \forall x, y \in H .
$$

And $z=P_{C} x$ if and only if there holds

$$
\langle x-z, y-z\rangle \leq 0, \quad \forall y \in C,
$$

and if and only if there holds the relation

$$
\|x-z\|^{2} \leq\|x-y\|^{2}-\|y-z\|^{2}, \quad \forall y \in C .
$$

Lemma 4 (see [9], Demiclosedness princple). Let $C$ be $a$ nonempty closed convex subset of a real Hilbert space $H$ and let $T: C \mapsto C$ be a nonexpansive mapping with $F(T) \neq \emptyset$. If $\left\{x_{n}\right\}$ is a sequence in $C$ weakly converging to $x$ and if $\left\{(I-T) x_{n}\right\}$ converges strongly to $y$, then $(I-T) x=y$; in particular if $y=0$, then $x \in F(T)$.

Lemma 5 (see [14]). Let $\lambda$ be a number in $[0,1]$ and $\mu \geq 0$. Let $F: H \mapsto H$ be a $t$-Lipschitzian and $\eta$-strongly monotone operator on a Hilbert space. Associate with a nonexpansive mapping $T: H \mapsto H$ and define the mapping $T^{\lambda}: H \mapsto H$ by

$$
T^{\lambda} x:=T x-\lambda \mu F(T x), \quad \forall x \in H .
$$

Then $T^{\lambda}$ is a contraction provided $\mu \leq 2 \eta / t^{2}$; that is,

$$
\left\|T^{\lambda} x-T^{\lambda} y\right\| \leq\left[1-\lambda \mu\left(\eta-\frac{\mu t^{2}}{2}\right)\right]\|x-y\|, \quad \forall x, y \in H .
$$

Lemma 6 (see [4]). Assume that $A$ is a strongly positive bounded linear operator on a Hilbert space $H$ with coefficient $\bar{\gamma}>0$ and $0<\rho \leq\|A\|^{-1}$; then $\|I-\rho A\| \leq 1-\rho \bar{\gamma}$. 
Lemma 7 (see [15]). Let C be a nonempty closed convex subset of a real Hilbert space $H$. Let $T: C \mapsto C$ be a $k$-strict pseudo-contractive mapping. Let $\gamma$ and $\delta$ be two nonnegative real numbers such that $(\gamma+\delta) k \leq \gamma$; then

$$
\|\gamma(x-y)+\delta(T x-T y)\| \leq(\gamma+\delta)\|x-y\|, \quad \forall x, y \in C .
$$

Lemma 8 (see [16]). Let $H$ be a Hilbert space and $C$ a nonempty convex subset of $H$. Let $T: C \mapsto H$ be a $k$-strict pseudo-contractive mapping. Define a mapping $J x=\delta x+(1-$ $\delta)$ Tx for all $x \in C$. Then as $\delta \in[k, 1)$, $J$ is a nonexpansive mapping such that $F(J)=F(T)$.

Lemma 9 (see [17]). Let $\left\{\alpha_{n}\right\}$ be a sequence of nonnegative real numbers satisfying the following relation: $\alpha_{n+1} \leq\left(1-\gamma_{n}\right) \alpha_{n}+\delta_{n}$, where (i) $\left\{\gamma_{n}\right\} \subset(0,1), \sum_{n=1}^{\infty} \gamma_{n}=\infty$; (ii) $\lim \sup _{n \rightarrow \infty}\left(\delta_{n} / \gamma_{n}\right)=$ 0 or $\sum_{n=1}^{\infty}\left|\delta_{n}\right|<\infty$; then $\lim _{n \rightarrow \infty} \alpha_{n}=0$.

\section{Main Results}

In this section, we prove the strong convergence results on the iterative algorithm for $k$-strict pseudo-contractions.

Theorem 10. Let $C$ be a nonempty closed convex subset of a real Hilbert space $H, S: C \mapsto H$ a non-self-L-Lipschitzian mapping, and $T: C \mapsto C$ a $k$-strict pseudo-contractive mapping such that $\operatorname{Fix}(T) \neq \emptyset$. Let $F: C \mapsto H$ be a $t$ Lipschitzian and $\eta$-strongly monotone mapping and $A: C \mapsto$ $C$ a $\bar{\gamma}$-strongly positive bounded linear operator. For a given $x_{0} \in C$, let the sequences $\left\{x_{n}\right\}$ and $\left\{y_{n}\right\}$ generated by (11), where $\left\{\alpha_{n}\right\},\left\{\gamma_{n}\right\},\left\{\delta_{n}\right\} \in[0,1]$, satisfy the following conditions:

(i) $\left[1-\mu\left(\eta-\mu t^{2} / 2\right)\right]((1+k) /(1-k)) \leq 1, \mu\left(\eta-\mu t^{2} / 2\right)-\tau L>$ $0, \bar{\gamma} \in(1,2)$

(ii) $\lim _{n \rightarrow \infty} \gamma_{n}=0, \lim _{n \rightarrow \infty} \delta_{n}=0, \sum_{n=0}^{\infty} \gamma_{n}=\infty, \sum_{n=0}^{\infty}$ $\delta_{n}=\infty,\left(\gamma_{n}+\delta_{n}\right) k \leq \gamma_{n}$

(iii) $\lim _{n \rightarrow \infty}\left(\alpha_{n} /\left(\gamma_{n}+\delta_{n}\right)\right)=0, \sum_{n=1}^{\infty}\left|\alpha_{n}-\alpha_{n-1}\right|<\infty$, $\sum_{n=1}^{\infty}\left|\gamma_{n}-\gamma_{n-1}\right|<\infty, \sum_{n=1}^{\infty}\left|\delta_{n}-\delta_{n-1}\right|<\infty$.

Then the sequence $\left\{x_{n}\right\}$ converges strongly to a fixed point $x^{*}$ of $T$, which solves the variational inequality

$$
\left\langle(I-A) x^{*}, z-x^{*}\right\rangle \leq 0, \quad \forall z \in \operatorname{Fix}(T) .
$$

Proof. The proof is divided into five steps.

Step 1. We first show that the sequences $\left\{x_{n}\right\},\left\{y_{n}\right\}$ are bounded. Take $p \in \operatorname{Fix}(T)$, own to $T: C \mapsto C$ be a $k$-strict pseudo-contractive mapping, and define $J x=k x+(1-k) T x$. By Lemma $8 J$ is nonexpansive and $\operatorname{Fix}(J)=\operatorname{Fix}(T)$; therefore $T x=(1 /(1-k))(J x-k x)$ :

$$
\begin{aligned}
& \left\|T x_{n}-T p\right\| \\
& =\left\|\frac{1}{1-k}\left(J x_{n}-k x_{n}\right)-\frac{1}{1-k}(J p-k p)\right\| \\
& =\frac{1}{1-k}\left\|\left(J x_{n}-J p\right)-k\left(x_{n}-p\right)\right\| \\
& \leq \frac{1+k}{1-k}\left\|x_{n}-p\right\| .
\end{aligned}
$$

Thus we immediately get that $T$ is a $(1+k) /(1-k)$ Lipschitzian mapping. Then we estimate $\left\|y_{n}-p\right\|$ :

$$
\begin{aligned}
&\left\|y_{n}-p\right\| \\
&=\left\|P_{C}\left[\alpha_{n} \tau S x_{n}+\left(I-\mu \alpha_{n} F\right) T x_{n}\right]-P_{C} p\right\| \\
& \leq\left\|\alpha_{n} \tau S x_{n}+\left(I-\mu \alpha_{n} F\right) T x_{n}-p\right\| \\
&=\| \alpha_{n}\left(\tau S x_{n}-\mu F p\right)+\left(I-\mu \alpha_{n} F\right) T x_{n} \\
& \quad \quad-\left(I-\mu \alpha_{n} F\right) T p \| \\
& \leq {\left[1-\mu\left(\eta-\frac{\mu t^{2}}{2}\right)\right] \frac{1+k}{1-k}\left\|x_{n}-p\right\| } \\
&+\alpha_{n}\left\|\tau S x_{n}-\mu F p\right\| \\
& \leq\left\|x_{n}-p\right\|+\alpha_{n}\left\|\tau S x_{n}-\mu F p\right\| .
\end{aligned}
$$

On the other hand, notice that $\lim _{n \rightarrow \infty} \gamma_{n}=0$, $\lim _{n \rightarrow \infty} \delta_{n}=0$; without loss of generality, we may assume that $\gamma_{n}+\delta_{n} \leq\|A\|^{-1}$; thus

$$
\begin{aligned}
&\left\|x_{n+1}-p\right\| \\
&=\left\|\left(I-\left(\gamma_{n}+\delta_{n}\right) A\right) y_{n}+\gamma_{n} x_{n}+\delta_{n} T x_{n}-p\right\| \\
& \leq\left\|\left(I-\left(\gamma_{n}+\delta_{n}\right) A\right) y_{n}-\left(I-\left(\gamma_{n}+\delta_{n}\right) A\right) p\right\| \\
&+\left\|\gamma_{n}\left(x_{n}-p\right)+\delta_{n}\left(T x_{n}-T p\right)\right\| \\
&+\left\|\left(\gamma_{n}+\delta_{n}\right)(I-A) p\right\| \\
& \leq {\left[1-\left(\gamma_{n}+\delta_{n}\right) \bar{\gamma}\right]\left\|y_{n}-p\right\| } \\
&+\left(\gamma_{n}+\delta_{n}\right)\left\|x_{n}-p\right\| \\
&+\left(\gamma_{n}+\delta_{n}\right)\|(I-A)\| \cdot\|p\| .
\end{aligned}
$$

Together with (24), we have

$$
\begin{aligned}
& \left\|x_{n+1}-p\right\| \\
& \leq\left[1-\left(\gamma_{n}+\delta_{n}\right)(\bar{\gamma}-1)\right]\left\|x_{n}-p\right\| \\
& +\alpha_{n}\left\|\tau S x_{n}-\mu F p\right\|+\left(\gamma_{n}+\delta_{n}\right)\|(I-A)\| \cdot\|p\| \\
& =\left[1-\left(\gamma_{n}+\delta_{n}\right)(\bar{\gamma}-1)\right]\left\|x_{n}-p\right\| \\
& +\left(\gamma_{n}+\delta_{n}\right)(\bar{\gamma}-1)\left[\frac{1}{\left(\gamma_{n}+\delta_{n}\right)(\bar{\gamma}-1)}\right. \\
& \times\left(\alpha_{n}\left\|\tau S x_{n}-\mu F p\right\|\right. \\
& \left.+\left(\gamma_{n}+\delta_{n}\right)\|(I-A)\| \cdot\|p\|\right] \\
& \leq \max \left\{\left\|x_{n}-p\right\|, \frac{1}{\bar{\gamma}-1}\left(\frac{\alpha_{n}}{\gamma_{n}+\delta_{n}}\left\|\tau S x_{n}-\mu F p\right\|\right.\right. \\
& +\|(I-A)\| \cdot\|p\|)\} .
\end{aligned}
$$


By conditions (ii) and (iii), we get that $\left\{x_{n}\right\}$ is bounded, and so are $\left\{y_{n}\right\},\left\{S x_{n}\right\},\left\{T x_{n}\right\},\left\{F T x_{n}\right\}$.

Step 2. Now we prove that $\left\|x_{n+1}-x_{n}\right\| \rightarrow 0$ as $n \rightarrow \infty$. Denote $\xi=\mu\left(\eta-\left(\mu t^{2} / 2\right)\right)$ :

$$
\begin{aligned}
\| y_{n}- & y_{n-1} \| \\
= & \| P_{C}\left[\alpha_{n} \tau S x_{n}+\left(I-\mu \alpha_{n} F\right) T x_{n}\right] \\
& -P_{C}\left[\alpha_{n-1} \tau S x_{n-1}+\left(I-\mu \alpha_{n-1} F\right) T x_{n-1}\right] \| \\
\leq & \| \alpha_{n} \tau S x_{n}+\left(I-\mu \alpha_{n} F\right) T x_{n} \\
& -\left[\alpha_{n-1} \tau S x_{n-1}+\left(I-\mu \alpha_{n-1} F\right) T x_{n-1}\right] \| \\
= & \| \alpha_{n} \tau\left(S x_{n}-S x_{n-1}\right)+\tau\left(\alpha_{n}-\alpha_{n-1}\right) S x_{n-1} \\
& +\left(I-\mu \alpha_{n} F\right) T x_{n}-\left(I-\mu \alpha_{n} F\right) T x_{n-1} \\
& -\mu\left(\alpha_{n}-\alpha_{n-1}\right) F T x_{n-1} \| \\
\leq & \alpha_{n} \tau L\left\|x_{n}-x_{n-1}\right\|+\left(1-\alpha_{n} \xi\right)\left\|x_{n}-x_{n-1}\right\| \\
& +\left|\alpha_{n}-\alpha_{n-1}\right|\left(\tau\left\|S x_{n-1}\right\|+\mu\left\|F T x_{n-1}\right\|\right) \\
= & {\left[1-\alpha_{n}(\xi-\tau L)\right]\left\|x_{n}-x_{n-1}\right\|+\left|\alpha_{n}-\alpha_{n-1}\right| M } \\
\leq & \left\|x_{n}-x_{n-1}\right\|+\left|\alpha_{n}-\alpha_{n-1}\right| M,
\end{aligned}
$$

where $M$ is a constant such that

$$
\begin{aligned}
\operatorname{Sup}\{ & \tau\left\|S x_{n-1}\right\|+\mu\left\|F T\left(x_{n-1}\right)\right\|+\left\|x_{n-1}\right\| \\
& \left.+\left\|T x_{n-1}\right\|+2\left\|A y_{n-1}\right\|\right\} \leq M, \\
\left\|x_{n+1}-x_{n}\right\| & \\
= & \|\left(I-\left(\gamma_{n}+\delta_{n}\right) A\right) y_{n}+\gamma_{n} x_{n}+\delta_{n} T x_{n} \\
& -\left[\left(I-\left(\gamma_{n-1}+\delta_{n-1}\right) A\right) y_{n-1}+\gamma_{n-1} x_{n-1}+\delta_{n-1} T x_{n-1}\right] \| \\
= & \|\left(I-\left(\gamma_{n}+\delta_{n}\right) A\right) y_{n}-\left(I-\left(\gamma_{n}+\delta_{n}\right) A\right) y_{n-1} \\
& +\left(\gamma_{n-1}-\gamma_{n}+\delta_{n-1}-\delta_{n}\right) A y_{n-1}+\gamma_{n}\left(x_{n}-x_{n-1}\right) \\
& +\delta_{n}\left(T x_{n}-T x_{n-1}\right)+\left(\gamma_{n}-\gamma_{n-1}\right) x_{n-1} \\
& +\left(\delta_{n}-\delta_{n-1}\right) T x_{n-1} \| \\
\leq & {\left[1-\left(\gamma_{n}+\delta_{n}\right) \bar{\gamma}\right]\left\|y_{n}-y_{n-1}\right\|+\left(\gamma_{n}+\delta_{n}\right)\left\|x_{n}-x_{n-1}\right\| } \\
& +\left(\left|\gamma_{n}-\gamma_{n-1}\right|+\left|\delta_{n}-\delta_{n-1}\right|\right)\left\|A y_{n-1}\right\| \\
& +\left|\gamma_{n}-\gamma_{n-1}\right|\left\|x_{n-1}\right\|+\left|\delta_{n}-\delta_{n-1}\right|\left\|T x_{n-1}\right\| \\
\leq & {\left[1-\left(\gamma_{n}+\delta_{n}\right) \bar{\gamma}\right]\left\|x_{n}-x_{n-1}\right\|+\left|\alpha_{n}-\alpha_{n-1}\right| M } \\
& +\left(\gamma_{n}+\delta_{n}\right)\left\|x_{n}-x_{n-1}\right\|+\left|\gamma_{n}-\gamma_{n-1}\right|\left(\left\|x_{n-1}\right\|+\left\|A y_{n-1}\right\|\right) \\
& +\left|\delta_{n}-\delta_{n-1}\right|\left(\left\|T x_{n-1}\right\|+\left\|A y_{n-1}\right\|\right) \\
\leq & {\left[1-\left(\gamma_{n}+\delta_{n}\right)(\bar{\gamma}-1)\right]\left\|x_{n}-x_{n-1}\right\| } \\
& M\left(\left|\alpha_{n}-\alpha_{n-1}\right|+\left|\gamma_{n}-\gamma_{n-1}\right|+\left|\delta_{n}-\delta_{n-1}\right|\right) . \\
&
\end{aligned}
$$

Thus we have $\left\|x_{n+1}-T x_{n}\right\| \rightarrow 0$ as $n \rightarrow \infty$. Observe that

$$
\left\|x_{n}-T x_{n}\right\| \leq\left\|x_{n}-x_{n+1}\right\|+\left\|x_{n+1}-T x_{n}\right\|
$$

we immediately get $\left\|x_{n}-T x_{n}\right\| \rightarrow 0$ as $n \rightarrow \infty$.

Step 4. Now we show that $\lim _{\sup _{n \rightarrow \infty}}\left\langle(I-A) x^{*}, x_{n}-x^{*}\right\rangle \leq$ 0 , where $x^{*} \in \operatorname{Fix}(T)$ is the unique solution of the variational inequality. Take a subsequence $\left\{x_{n_{k}}\right\}$ of $\left\{x_{n}\right\}$ such that

$$
\begin{aligned}
& \limsup _{n \rightarrow \infty}\left\langle(I-A) x^{*}, x_{n}-x^{*}\right\rangle \\
& =\lim _{k \rightarrow \infty}\left\langle(I-A) x^{*}, x_{n_{k}}-x^{*}\right\rangle .
\end{aligned}
$$

Observe that the sequence $\left\{x_{n}\right\}$ is bounded; without loss of generality we may assume that $x_{n_{k}} \rightarrow x^{\prime}$. By Lemma 4 , we get $x^{\prime} \in \operatorname{Fix}(T)$. Therefore by Lemma 3, we have

$$
\begin{aligned}
& \limsup _{n \rightarrow \infty}\left\langle(I-A) x^{*}, x_{n}-x^{*}\right\rangle \\
& =\left\langle(I-A) x^{*}, x^{\prime}-x^{*}\right\rangle \leq 0 .
\end{aligned}
$$


Step 5. Next we prove that $\left\|x_{n+1}-x^{*}\right\| \rightarrow 0$ as $n \rightarrow \infty$ :

$$
\begin{aligned}
\| x_{n+1} & -x^{*} \|^{2} \\
= & \|\left(I-\left(\gamma_{n}+\delta_{n}\right) A\right) y_{n} \\
& -\left(I-\left(\gamma_{n}+\delta_{n}\right) A\right) x^{*}+\gamma_{n}\left(x_{n}-x^{*}\right) \\
& +\delta_{n}\left(T x_{n}-T x^{*}\right)+\left(\gamma_{n}+\delta_{n}\right)(I-A) x^{*} \|^{2} \\
\leq & \left\|\left(I-\left(\gamma_{n}+\delta_{n}\right) A\right)\left(y_{n}-x^{*}\right)\right\|^{2} \\
& +2\left\langle\gamma_{n}\left(x_{n}-x^{*}\right)+\delta_{n}\left(T x_{n}-T x^{*}\right), x_{n+1}-x^{*}\right\rangle \\
& +2\left\langle\left(\gamma_{n}+\delta_{n}\right)(I-A) x^{*}, x_{n+1}-x^{*}\right\rangle \\
\leq & {\left[1-\left(\gamma_{n}+\delta_{n}\right) \bar{\gamma}\right]^{2}\left\|y_{n}-x^{*}\right\|^{2} } \\
& +2\left\|\gamma_{n}\left(x_{n}-x^{*}\right)+\delta_{n}\left(T x_{n}-T x^{*}\right)\right\| \cdot\left\|x_{n+1}-x^{*}\right\| \\
& +2\left(\gamma_{n}+\delta_{n}\right)\left\langle(I-A) x^{*}, x_{n+1}-x^{*}\right\rangle \\
\leq & \left\|1-\left(\gamma_{n}+\delta_{n}\right) \bar{\gamma}\right\|^{2}\left\|y_{n}-x^{*}\right\|^{2} \\
& +2\left(\gamma_{n}+\delta_{n}\right)\left\|x_{n}-x^{*}\right\| \cdot\left\|x_{n+1}-x^{*}\right\| \\
& +2\left(\gamma_{n}+\delta_{n}\right)\left\langle(I-A) x^{*}, x_{n+1}-x^{*}\right\rangle .
\end{aligned}
$$

Notice that

$$
\begin{aligned}
& \left\|y_{n}-x^{*}\right\|^{2} \leq\left\|x_{n}-x^{*}\right\|^{2} \\
& +2 \alpha_{n}\left\|x_{n}-x^{*}\right\| \cdot\left\|\tau S x_{n}-\mu F x^{*}\right\| \\
& +\alpha_{n}^{2}\left\|\tau S x_{n}-\mu F x^{*}\right\|^{2}
\end{aligned}
$$

thus

$$
\begin{aligned}
\left\|x_{n+1}-x^{*}\right\|^{2} & \\
\leq & {\left[1-\left(\gamma_{n}+\delta_{n}\right) \bar{\gamma}\right]^{2} } \\
\times & {\left[\left\|x_{n}-x^{*}\right\|^{2}+2 \alpha_{n}\left\|x_{n}-x^{*}\right\|\right.} \\
& \left.\cdot\left\|\tau S x_{n}-\mu F x^{*}\right\|+\alpha_{n}^{2}\left\|\tau S x_{n}-\mu F x^{*}\right\|^{2}\right] \\
\times & \left(\gamma_{n}+\delta_{n}\right)\left(\left\|x_{n}-x^{*}\right\|^{2}+\left\|x_{n+1}-x^{*}\right\|^{2}\right) \\
+ & 2\left(\gamma_{n}+\delta_{n}\right)\left\langle(I-A) x^{*}, x_{n+1}-x^{*}\right\rangle . \\
{[1-} & \left.\left(\gamma_{n}+\delta_{n}\right)\right]\left\|x_{n+1}-x^{*}\right\|^{2} \\
\leq & {\left[1-\left(\gamma_{n}+\delta_{n}\right) \bar{\gamma}\right]^{2}\left\|x_{n}-x^{*}\right\|^{2} }
\end{aligned}
$$

$$
\begin{aligned}
& +\left(\gamma_{n}+\delta_{n}\right)\left\|x_{n}-x^{*}\right\|^{2} \\
& +\left[2 \alpha_{n}\left\|x_{n}-x^{*}\right\| \cdot\left\|\tau S x_{n}-\mu F x^{*}\right\|\right. \\
& +\alpha_{n}^{2}\left\|\tau S x_{n}-\mu F x^{*}\right\|^{2} \\
& \left.+2\left(\gamma_{n}+\delta_{n}\right)\left\langle(I-A) x^{*}, x_{n+1}-x^{*}\right\rangle\right], \\
& \left\|x_{n+1}-x^{*}\right\|^{2}=\left[1-\frac{2\left(\gamma_{n}+\delta_{n}\right)(\bar{\gamma}-1)}{1-\left(\gamma_{n}+\delta_{n}\right)}\right]\left\|x_{n}-x^{*}\right\|^{2} \\
& +\frac{1}{1-\left(\gamma_{n}+\delta_{n}\right)} \\
& \times\left[\left(\gamma_{n}+\delta_{n}\right)^{2} \bar{\gamma}^{2}\left\|x_{n}-x^{*}\right\|^{2}\right. \\
& +2 \alpha_{n}\left\|x_{n}-x^{*}\right\| \cdot\left\|\tau S x_{n}-\mu F x^{*}\right\| \\
& +\alpha_{n}^{2}\left\|\tau S x_{n}-\mu F x^{*}\right\|^{2} \\
& \left.+2\left(\gamma_{n}+\delta_{n}\right)\left\langle(I-A) x^{*}, x_{n+1}-x^{*}\right\rangle\right] \\
& =\left[1-\frac{2\left(\gamma_{n}+\delta_{n}\right)(\bar{\gamma}-1)}{1-\left(\gamma_{n}+\delta_{n}\right)}\right]\left\|x_{n}-x^{*}\right\|^{2} \\
& +\frac{2\left(\gamma_{n}+\delta_{n}\right)(\bar{\gamma}-1)}{1-\left(\gamma_{n}+\delta_{n}\right)} \\
& \times\left\{\frac{1}{2\left(\gamma_{n}+\delta_{n}\right)(\bar{\gamma}-1)}\right. \\
& \times\left[\left(\gamma_{n}+\delta_{n}\right)^{2} \bar{\gamma}^{2}\left\|x_{n}-x^{*}\right\|^{2}\right. \\
& +2 \alpha_{n}\left\|x_{n}-x^{*}\right\| \cdot\left\|\tau S x_{n}-\mu F x^{*}\right\| \\
& +\alpha_{n}^{2}\left\|\tau S x_{n}-\mu F x^{*}\right\|^{2}+2\left(\gamma_{n}+\delta_{n}\right) \\
& \left.\left.\times\left\langle(I-A) x^{*}, x_{n+1}-x^{*}\right\rangle\right]\right\} \text {. }
\end{aligned}
$$

By the conditions (ii), (iii) and Lemma 9, we conclude that $\left\|x_{n}-x^{*}\right\| \rightarrow 0$ as $n \rightarrow \infty$, which solves the variational inequality $\left\langle(I-A) x^{*}, z-x^{*}\right\rangle \leq 0$, for all $z \in \operatorname{Fix}(T)$. This completes the proof.

Remark 11. The iterative algorithm in Theorem 10 here is a new approximating method, and Lemma 7 plays a key role in the proof of the main results which makes the proof simple.

Remark 12. The results in this paper improve and extend some recent related results. For example, Theorem 10 here improves and extends Theorem 3.2 in [8] in the following ways:

(i) the nonexpansive mapping $T: C \mapsto C$ in $[8]$ is extended to the case of $k$-strict pseudo-contractions $T: C \mapsto C$; 
(ii) the self-contraction $f: C \mapsto C$ in [8] is extended to the case of a (possiblly non-self) Lipschitzian mapping $S: C \mapsto H$.

\section{Acknowledgments}

The authors would like to thank editors and referees for many useful comments and suggestions for the improvement of the paper. This work is partially supported by the Natural Science Foundation of Zhejiang Province (Y6100696, Y6110270) and the National Natural Science Foundation $(11071169,11271330)$.

\section{References}

[1] A. Moudafi, "Viscosity approximation methods for fixed-points problems," Journal of Mathematical Analysis and Applications, vol. 241, no. 1, pp. 46-55, 2000.

[2] H.-K. Xu, "Viscosity approximation methods for nonexpansive mappings," Journal of Mathematical Analysis and Applications, vol. 298, no. 1, pp. 279-291, 2004.

[3] M. Tian, "A general iterative algorithm for nonexpansive mappings in Hilbert spaces," Nonlinear Analysis: Theory, Methods \& Application A, vol. 73, no. 3, pp. 689-694, 2010.

[4] G. Marino and H.-K. Xu, "A general iterative method for nonexpansive mappings in Hilbert spaces," Journal of Mathematical Analysis and Applications, vol. 318, no. 1, pp. 43-52, 2006.

[5] F. Deutsch and I. Yamada, "Minimizing certain convex functions over the intersection of the fixed point sets of nonexpansive mappings," Numerical Functional Analysis and Optimization, vol. 19, no. 1-2, pp. 33-56, 1998.

[6] H. K. Xu, "An iterative approach to quadratic optimization," Journal of Optimization Theory and Applications, vol. 116, no. 3, pp. 659-678, 2003.

[7] I. Yamada, "The hybrid steepest descent method for the variational inequality problem over the intersection of fixed point sets of nonexpansive mappings," in Inherently Parallel Algorithms in Feasibility and Optimization and Their Applications, D. Butnariu, Y. Censor, and S. ReichS, Eds., vol. 8, pp. 473-504, North-Holland, Amsterdam, The Netherland, 2001.

[8] L.-C. Ceng, S.-M. Guu, and J.-C. Yao, "A general composite iterative algorithm for nonexpansive mappings in Hilbert spaces," Computers \& Mathematics with Applications, vol. 61, no. 9, pp. 2447-2455, 2011.

[9] Y. H. Wang and Y. H. Xia, "Strong convergence for asymptotically pseudo-contractions with the demiclosedness principle in Banach spaces," Fixed Point Theory and Applications, vol. 2012, article 45, 2012.

[10] Y. L. Song, H. Y. Hu, Y. Q. Wang et al., "Strong convergence of a new general iterative method for variational inequality problems in Hilbert spaces," Fixed Point Theory and Applications, vol. 2012, article 46, 2012.

[11] W. Xu and Y. H. Wang, "Strong convergence of the iterative methods for hierarchical fixed point problems of an infinite family strictly non-self pseudo-contractions," Abstract and Applied Analysis, vol. 2012, Article ID 457024, 11 pages, 2012.

[12] N. Young, An Introduction to Hilbert Space, Cambridge University Press, Cambridge, UK, 1988.

[13] Y. H. Yao, M. A. Noor, and Y. C. Liou, "Strong convergence of a modified extragradient method to the miniumnorm solution of variational inequalities," Abstract and Applied Analysis, vol. 2012, Article ID 817436, 9 pages, 2012.

[14] H. K. Xu and T. H. Kim, "Convergence of hybrid steepestdescent methods for variational inequalities," Journal of Optimization Theory and Applications, vol. 119, no. 1, pp. 185-201, 2003.

[15] Y. Yao, Y.-C. Liou, and S. M. Kang, "Approach to common elements of variational inequality problems and fixed point problems via a relaxed extragradient method," Computers \& Mathematics with Applications, vol. 59, no. 11, pp. 3472-3480, 2010.

[16] F. E. Browder and W. V. Petryshyn, "Construction of fixed points of nonlinear mappings in Hilbert space," Journal of Mathematical Analysis and Applications, vol. 20, pp. 197-228, 1967.

[17] Y. Wang and L. Yang, "Modified relaxed extragradient method for a general system of variational inequalities and nonexpansive mappings in Banach spaces," Abstract and Applied Analysis, vol. 2012, Article ID 818970, 14 pages, 2012. 


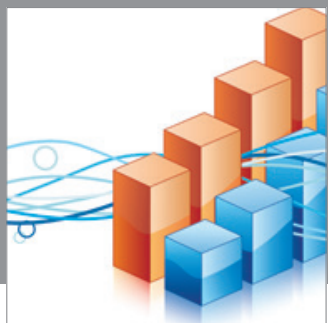

Advances in

Operations Research

mansans

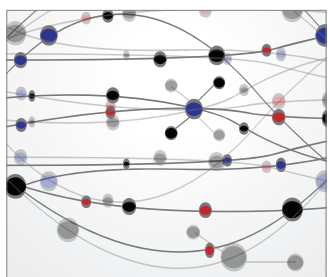

The Scientific World Journal
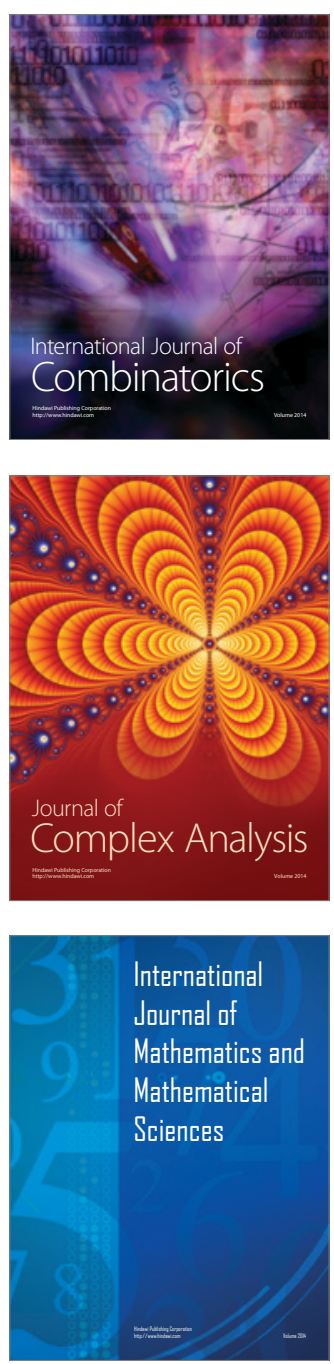
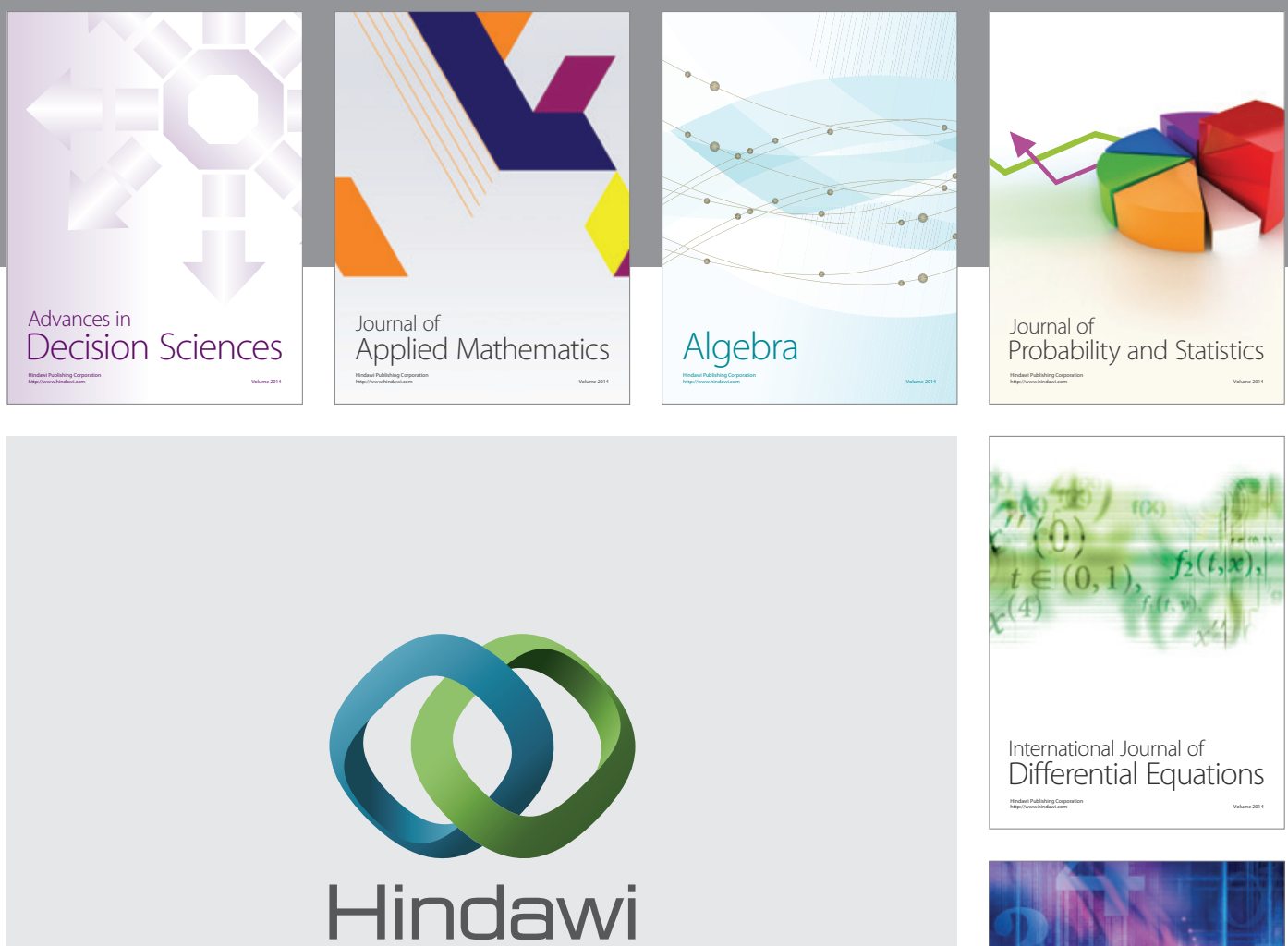

Submit your manuscripts at http://www.hindawi.com
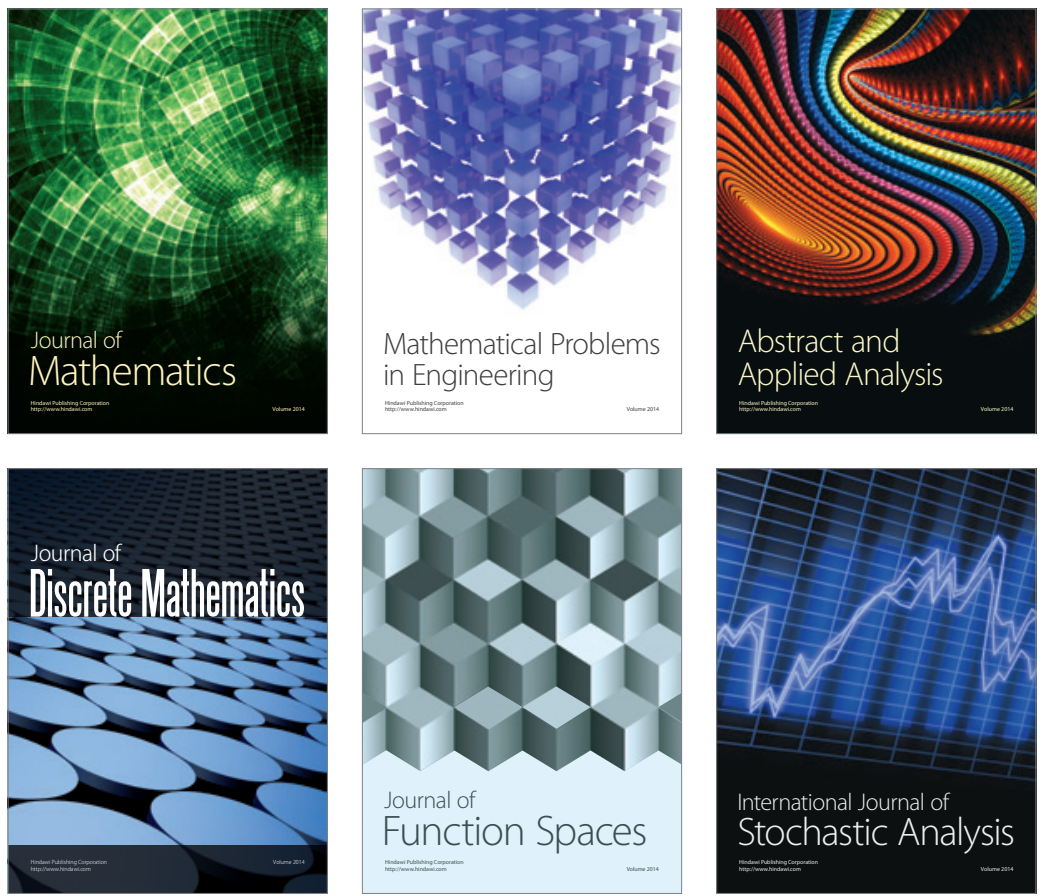

Journal of

Function Spaces

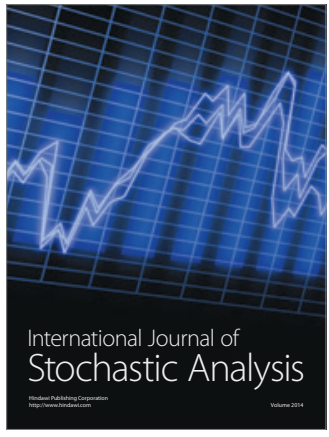

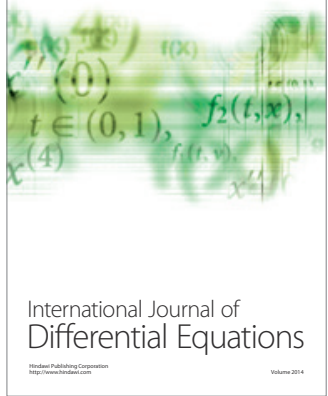
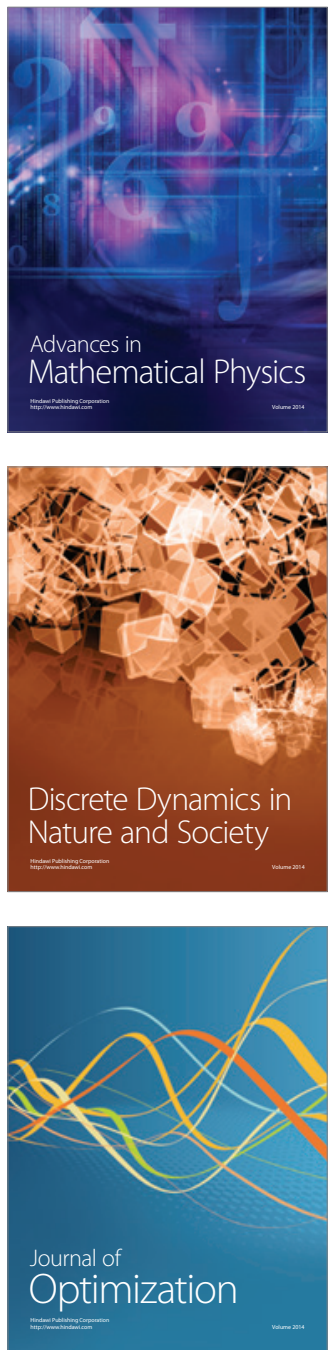